\title{
Phase Transformations of Azithromycin Crystals Investigated by Thermal and Spectroscopic Analyses Combined with ab Initio Calculations
}

Naiane S. Santana ${ }^{\dagger}$, Anderson F. Gomes ${ }^{\S}$, H. S. Santana ${ }^{\perp}$, Gilberto D. Saraiva ${ }^{\ddagger}$, Paulo R. S. Ribeiro ${ }^{\S}$, Adenilson O. Santos ${ }^{\S}$, Carlos E. S. Nogueira ${ }^{\perp}$, and Francisco F. de Sousa ${ }^{*}+, \S$

†Instituto de Ciências Exatas e Naturais, Universidade Federal do Pará, CEP 66075-110, Belém, PA, Brazil ${ }^{\S}$ Programa de Pós-Graduação em Ciência dos Materiais, CCSST, Universidade Federal do Maranhão, CEP 65900-410, Imperatriz, MA, Brazil

${ }^{\perp}$ Departamento de Física, Universidade Regional do Cariri, CEP 63010-970, Juazeiro do Norte, CE, Brazil *Faculdade de Educação Ciências e Letras do Sertão Central, Universidade Estadual do Ceará, CEP 63900000, Quixadá, CE, Brazil

\section{SUPPORTING INFORMATION}

\section{Contents:}

Calculated vibrational wavenumbers unscaled and scaled by the scale factors 0.9630 (20 to 2000 $\mathrm{cm}^{-1}$ ) and 0.940 (2500 to $4000 \mathrm{~cm}^{-1}$ ), experimental Raman and absorbance band positions and assignment of vibrational modes of orthorhombic AZM-DH crystal - Table S1.

Table S1.

\begin{tabular}{|c|c|c|c|c|}
\hline$\omega_{\text {calc }}$ & $\omega_{\text {scal }}$ & $\omega_{\text {Raman }}$ & $\omega_{\text {IR }}$ & Assignment with PED ${ }^{*}$ [\%] \\
\hline 3762 & 3536 & $3557 \mathrm{vw}$ & 3560 & $v(\mathrm{O} 49 \mathrm{H} 124)[100]$ \\
\hline 3739 & 3515 & 3496vw & 3490 & $v(\mathrm{O} 13 \mathrm{H} 122)[100]$ \\
\hline 3643 & 3424 & & 3420 & $v(\mathrm{O} 27 \mathrm{H} 83)[96]$ \\
\hline 3615 & 3398 & & & $v(\mathrm{O} 24 \mathrm{H} 123)[95]$ \\
\hline 3308 & 3110 & 3111vw & 3100 & $v(\mathrm{O} 13 \mathrm{H} 63)[99]$ \\
\hline 3182 & 2991 & 3010w & 2993 & $\mathrm{v}_{\mathrm{as}}\left(\mathrm{C}_{2} \mathrm{H}_{2}\right)[98]$ \\
\hline 3166 & 2976 & $2979 m$ & 2979 & $v(\mathrm{C} 9 \mathrm{H} 60)[86]$ \\
\hline 3162 & 2972 & $2971 \mathrm{~m}$ & 2971 & $v(\mathrm{C} 14 \mathrm{H} 65)[81]$ \\
\hline 3158 & 2969 & & & $v_{\mathrm{as}}\left(\mathrm{C} 22 \mathrm{H}_{2}\right)[94]$ \\
\hline 3156 & 2967 & & & $\mathrm{v}_{\mathrm{as}}\left(\mathrm{C} 45 \mathrm{H}_{2}\right)[92]$ \\
\hline 3154 & 2965 & & & $\mathrm{v}_{\mathrm{as}}\left(\mathrm{C} 5 \mathrm{H}_{2}\right)[96]$ \\
\hline 3151 & 2962 & & & $v_{\mathrm{as}}\left(\mathrm{C} 26 \mathrm{H}_{2}\right)[89]$ \\
\hline 3144 & 2955 & & & $v(\mathrm{C} 47 \mathrm{H} 114)[89]$ \\
\hline 3143 & 2954 & $2955 \mathrm{~m}$ & 2958 & $\mathrm{v}_{\mathrm{as}}\left(\mathrm{C} 26 \mathrm{H}_{2}\right)[94]$ \\
\hline
\end{tabular}




\begin{tabular}{|c|c|c|c|c|}
\hline 3138 & 2950 & & & $v(\mathrm{C} 51 \mathrm{H} 119)[74]$ \\
\hline 3134 & 2946 & & & $v_{\text {as }}\left(\mathrm{C}_{22} \mathrm{H}_{3}\right)[98]$ \\
\hline 3132 & 2944 & & & $v_{\mathrm{as}}\left(\mathrm{C}_{5} \mathrm{H}_{2}\right)[74]$ \\
\hline 3132 & 2944 & & & $v(\mathrm{C} 40 \mathrm{H} 105)[26]+v(\mathrm{C} 50 \mathrm{H} 118)[26]+v(\mathrm{C} 51 \mathrm{H} 120)[20]$ \\
\hline 3129 & 2941 & & & 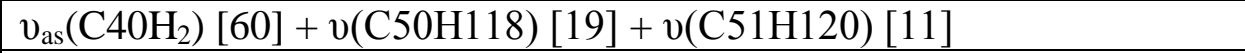 \\
\hline 3127 & 2939 & & & $\mathrm{v}_{\mathrm{as}}\left(\mathrm{C}_{2} 9 \mathrm{H}_{2}\right)[43]+\mathrm{v}_{\mathrm{as}}\left(\mathrm{C}_{3} \mathrm{HH}_{2}\right)[43]$ \\
\hline 3125 & 2938 & & & $v_{\text {as }}\left(\mathrm{C}_{40 H_{2}}\right)[89]$ \\
\hline 3124 & 2937 & & & $v_{\mathrm{as}}\left(\mathrm{C} 14 \mathrm{H}_{2}\right)[81]$ \\
\hline 3123 & 2936 & & & $v_{\mathrm{as}}\left(\mathrm{C}_{45 \mathrm{H}_{2}}\right)[90]$ \\
\hline 3120 & 2933 & $2933 \mathrm{vs}$ & 2934 & $v_{\mathrm{as}}\left(\mathrm{C}_{3} \mathrm{H}_{3}\right)[78]+v(\mathrm{C} 28 \mathrm{H} 87)[17]$ \\
\hline 3119 & 2932 & & & $v(\mathrm{C} 17 \mathrm{H} 72)[39]+v(\mathrm{C} 20 \mathrm{H} 77)[21]$ \\
\hline 3119 & 2932 & & & $\mathrm{v}_{\mathrm{as}}\left(\mathrm{C} 36 \mathrm{H}_{2}\right)[86]$ \\
\hline 3118 & 2931 & & & $v(\mathrm{C} 17 \mathrm{H} 72)[23]+v(\mathrm{C} 20 \mathrm{H} 77)[53]$ \\
\hline 3116 & 2929 & & & $v(\mathrm{C} 6 \mathrm{H} 57)[53]+v(\mathrm{C} 10 \mathrm{H} 62)[18]$ \\
\hline 3115 & 2928 & & & $v_{\mathrm{as}}\left(\mathrm{C}_{\mathrm{H}} \mathrm{H}_{2}\right)[62]+v(\mathrm{C} 9 \mathrm{H} 61)[36]+\mathrm{v}(\mathrm{C} 10 \mathrm{H} 62)[14]$ \\
\hline 3114 & 2927 & & & $v(\mathrm{C} 43 \mathrm{H} 2)[76]$ \\
\hline 3112 & 2925 & & & $v(\mathrm{C} 6 \mathrm{H} 57)[14]+v(\mathrm{C} 43 \mathrm{H} 109)[17]+v(\mathrm{C} 50 \mathrm{H} 118)[24]+v_{\mathrm{as}}\left(\mathrm{C} 51 \mathrm{H}_{2}\right)[25]$ \\
\hline 3112 & 2925 & & & $v(\mathrm{C} 28 \mathrm{H} 87)[62]+v_{\mathrm{as}}\left(\mathrm{C}_{3} \mathrm{HH}_{2}\right)[28]$ \\
\hline 3110 & 2923 & & & $v_{a s}\left(C 37 H_{2}\right)[96]$ \\
\hline 3109 & 2922 & & & $v(\mathrm{C} 6 \mathrm{H} 57)[18]+v(\mathrm{C} 10 \mathrm{H} 62)[54]$ \\
\hline 3107 & 2921 & & & $v(\mathrm{C} 15 \mathrm{H} 67)[83]$ \\
\hline 3103 & 2917 & & & $v(\mathrm{C} 29 \mathrm{H} 88)[39]+v_{\mathrm{as}}\left(\mathrm{C}_{30 \mathrm{H}_{2}}\right)[35]$ \\
\hline 3103 & 2917 & & & $v_{\mathrm{as}}\left(\mathrm{C}_{\left.17 \mathrm{H}_{2}\right)}\right)[53]+\mathrm{v}(\mathrm{C} 23 \mathrm{H} 82)[12]$ \\
\hline 3101 & 2915 & & & $v_{a s}\left(\mathrm{C}_{17 H_{2}}\right)[27]+v(\mathrm{C} 23 \mathrm{H} 82)[54]$ \\
\hline 3101 & 2915 & & & $v_{\mathrm{as}}\left(\mathrm{C}_{38 \mathrm{H}_{3}}\right)[91]$ \\
\hline 3100 & 2914 & $2911 \mathrm{~m}$ & 2912 & $v(\mathrm{C} 4 \mathrm{H} 53)[56]+v(\mathrm{C} 42 \mathrm{H} 108)[31]$ \\
\hline 3095 & 2909 & & & $v(\mathrm{C} 4 \mathrm{H} 53)[22]+v(\mathrm{C} 42 \mathrm{H} 108)[54]$ \\
\hline 3091 & 2906 & & & $v(\mathrm{C} 21 \mathrm{H} 78)[68]+v(\mathrm{C} 23 \mathrm{H} 82)[14]$ \\
\hline 3085 & 2900 & & & $v_{\text {as }}\left(\mathrm{C}_{4} 7 \mathrm{H}_{2}\right)[93]$ \\
\hline 3075 & 2891 & & & $v(\mathrm{C} 16 \mathrm{H} 69)[44]+v(\mathrm{C} 18 \mathrm{H} 74)[14]$ \\
\hline 3073 & 2889 & $2889 \mathrm{~m}$ & 2892 & $v_{s}\left(\mathrm{C}_{2} 6 \mathrm{H}_{3}\right)[84]$ \\
\hline 3069 & 2885 & & & $v(\mathrm{C} 36 \mathrm{H} 97)[93]$ \\
\hline 3069 & 2885 & & & $v_{\mathrm{s}}\left(\mathrm{C} 43 \mathrm{H}_{2}\right)[66]+v_{\mathrm{s}}\left(\mathrm{C} 45 \mathrm{H}_{2}\right)[20]$ \\
\hline 3065 & 2881 & & & $v_{\mathrm{as}}\left(\mathrm{C} 37 \mathrm{H}_{3}\right)[96]$ \\
\hline 3065 & 2881 & & & $v(\mathrm{C} 8 \mathrm{H} 58)[27]+v_{s}\left(\mathrm{C}^{2} \mathrm{H}_{2}\right)[32]$ \\
\hline 3064 & 2880 & & & $v_{s}\left(\mathrm{C}_{2} 2 \mathrm{H}_{3}\right)[77]$ \\
\hline 3063 & 2879 & & & $v_{s}\left(\mathrm{C}_{5} \mathrm{H}_{3}\right)[92]$ \\
\hline 3063 & 2879 & & & $v_{s}\left(\mathrm{C} 14 \mathrm{H}_{2}\right)[44]+v(\mathrm{C} 18 \mathrm{H} 74)[19]$ \\
\hline 3063 & 2879 & & & $v_{\mathrm{s}}\left(\mathrm{C}_{2} 9 \mathrm{H}_{2}\right)[92]$ \\
\hline 3060 & 2876 & & & $v_{s}\left(\mathrm{C}_{\left.45 \mathrm{H}_{3}\right)}\right)[68]+v(\mathrm{C} 43 \mathrm{H} 110)[20]$ \\
\hline 3060 & 2876 & & & $v(\mathrm{C} 8 \mathrm{H} 58)[62]+v_{\mathrm{s}}\left(\mathrm{C}_{9} \mathrm{H}_{2}\right)[29]$ \\
\hline 3056 & 2873 & & 2875 & 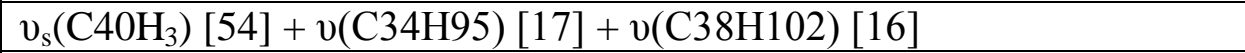 \\
\hline 3056 & 2873 & & & $v(\mathrm{C} 16 \mathrm{H} 69)[33]+v(\mathrm{C} 18 \mathrm{H} 74)[43]$ \\
\hline 3053 & 2870 & & & $v_{s}\left(\mathrm{C}_{40 H_{3}}\right)[43]+v(\mathrm{C} 34 \mathrm{H} 95)[34]$ \\
\hline 3053 & 2870 & & & $v_{s}\left(C_{51 H_{3}}\right)[98]$ \\
\hline 3049 & 2866 & & & $v_{s}\left(\mathrm{C}_{30 H_{3}}\right)[99]$ \\
\hline
\end{tabular}




\begin{tabular}{|c|c|c|c|c|}
\hline 3044 & 2861 & & & $v(\mathrm{C} 31 \mathrm{H} 93)[14]+v(\mathrm{C} 34 \mathrm{H} 95)[21]+v(\mathrm{C} 38 \mathrm{H} 102)[48]$ \\
\hline 3038 & 2856 & & & $v_{\mathrm{s}}\left(\mathrm{Cl}_{7} \mathrm{H}_{3}\right)[97]$ \\
\hline 3038 & 2856 & & & $v(\mathrm{C} 31 \mathrm{H} 93)[71]+v(\mathrm{C} 34 \mathrm{H} 95)[22]$ \\
\hline 3025 & 2844 & $2849 w$ & & $v_{s}\left(\mathrm{C}_{4} 7 \mathrm{H}_{2}\right)[89]$ \\
\hline 3024 & 2843 & & & $v(\mathrm{C} 15 \mathrm{H} 68)[91]$ \\
\hline 3019 & 2838 & $2837 \mathrm{w}$ & 2850 & $v(\mathrm{C} 39 \mathrm{H} 104)[93]$ \\
\hline 3008 & 2828 & $2828 \mathrm{w}$ & 2829 & $v(\mathrm{C} 32 \mathrm{H} 94)[80]$ \\
\hline 3003 & 2823 & & & $v_{s}\left(C 37 H_{3}\right)[89]$ \\
\hline 3001 & 2821 & & & $v(\mathrm{C} 20 \mathrm{H} 76)[92]$ \\
\hline 2990 & 2811 & & & $v(\mathrm{C} 48 \mathrm{H} 117)[94]$ \\
\hline 2960 & 2782 & $2782 \mathrm{w}$ & 2783 & $v(\mathrm{C} 18 \mathrm{H} 73)[95]$ \\
\hline 2959 & 2781 & & & $v(\mathrm{C} 36 \mathrm{H} 98)[93]$ \\
\hline 1776 & 1705 & $1719 \mathrm{w}$ & 1720 & $v(\mathrm{O} 3=\mathrm{C} 2)[87]$ \\
\hline 1554 & 1492 & & & $\delta(\mathrm{H} 81 \mathrm{C} 22 \mathrm{H} 80)[29]+\delta(\mathrm{H} 76 \mathrm{C} 20 \mathrm{H} 75)[19]+\delta(\mathrm{H} 79 \mathrm{C} 22 \mathrm{H} 81)[11]$ \\
\hline 1553 & 1491 & & & $\begin{array}{l}\delta(\mathrm{H} 96 \mathrm{C} 36 \mathrm{H} 98)[30]+\delta(\mathrm{H} 101 \mathrm{C} 37 \mathrm{H} 100)[13]+\delta(\mathrm{H} 98 \mathrm{C} 36 \mathrm{H} 97)[16]+ \\
\delta(\mathrm{H} 100 \mathrm{C} 37 \mathrm{H} 99)[19]\end{array}$ \\
\hline 1549 & 1487 & & & $\delta(\mathrm{H} 60 \mathrm{C} 9 \mathrm{H} 59)[42]+\delta(\mathrm{H} 61 \mathrm{C} 9 \mathrm{H} 60)[19]$ \\
\hline 1547 & 1485 & & & $\delta(\mathrm{H} 84 \mathrm{C} 26 \mathrm{H} 86)[10]+\delta(\mathrm{H} 85 \mathrm{C} 26 \mathrm{H} 84)[59]$ \\
\hline 1543 & 1481 & & & $\delta(\mathrm{H} 75 \mathrm{C} 20 \mathrm{H} 77)[24]+\delta(\mathrm{H} 77 \mathrm{C} 20 \mathrm{H} 76)[14]+\delta(\mathrm{H} 79 \mathrm{C} 22 \mathrm{H} 81)[11]$ \\
\hline 1543 & 1481 & & & $\delta(\mathrm{H} 114 \mathrm{C} 47 \mathrm{H} 116)[36]+\delta(\mathrm{H} 115 \mathrm{C} 47 \mathrm{H} 114)[26]$ \\
\hline 1542 & 1480 & & & $\delta(\mathrm{H} 54 \mathrm{C} 5 \mathrm{H} 56)[26]+\delta(\mathrm{H} 56 \mathrm{C} 5 \mathrm{H} 55)[37]$ \\
\hline 1539 & 1477 & & & $\begin{array}{l}\delta(\mathrm{H} 96 \mathrm{C} 36 \mathrm{H} 98)[19]+\delta(\mathrm{H} 101 \mathrm{C} 37 \mathrm{H} 100)[26]+\delta(\mathrm{H} 98 \mathrm{C} 36 \mathrm{H} 97)[10]+ \\
\delta(\mathrm{H} 100 \mathrm{C} 37 \mathrm{H} 99)[24]\end{array}$ \\
\hline 1539 & 1477 & & & $\begin{array}{l}\delta(\mathrm{H} 114 \mathrm{C} 47 \mathrm{H} 116)[11]+\delta(\mathrm{H} 113 \mathrm{C} 45 \mathrm{H} 112)[12]+\delta(\mathrm{H} 115 \mathrm{C} 47 \mathrm{H} 114)[16]+ \\
\delta(\mathrm{H} 116 \mathrm{C} 47 \mathrm{H} 115)[20]\end{array}$ \\
\hline 1537 & 1476 & & & $\delta(\mathrm{H} 90 \mathrm{C} 30 \mathrm{H} 92)[33]+\delta(\mathrm{H} 92 \mathrm{C} 30 \mathrm{H} 91)[26]$ \\
\hline 1536 & 1475 & & & $\delta(\mathrm{H} 77 \mathrm{C} 20 \mathrm{H} 76)[31]+\delta(\mathrm{H} 81 \mathrm{C} 22 \mathrm{H} 80)[15]$ \\
\hline 1535 & 1474 & & & $\delta(\mathrm{H} 59 \mathrm{C} 9 \mathrm{H} 61)[11]+\delta(\mathrm{H} 64 \mathrm{C} 14 \mathrm{H} 66)[11]+\delta(\mathrm{H} 71 \mathrm{C} 17 \mathrm{H} 70)[10]$ \\
\hline 1534 & 1473 & & & $\delta(\mathrm{H} 59 \mathrm{C} 9 \mathrm{H} 61)[27]+\delta(\mathrm{H} 61 \mathrm{C} 9 \mathrm{H} 60)[26]$ \\
\hline 1532 & 1471 & & & $\delta(\mathrm{H} 112 \mathrm{C} 45 \mathrm{H} 111)[20]+\delta(\mathrm{H} 116 \mathrm{C} 47 \mathrm{H} 115)[21]$ \\
\hline 1531 & 1470 & & & $\begin{array}{l}\delta(\mathrm{H} 71 \mathrm{C} 17 \mathrm{H} 70)[14]+\delta(\mathrm{H} 119 \mathrm{C} 51 \mathrm{H} 121)[25]+\delta(\mathrm{H} 120 \mathrm{C} 51 \mathrm{H} 119)[11]+ \\
\delta(\mathrm{H} 106 \mathrm{C} 40 \mathrm{H} 105)[11]\end{array}$ \\
\hline 1531 & 1470 & & & $\delta(\mathrm{H} 120 \mathrm{C} 51 \mathrm{H} 119)[12]+\delta(\mathrm{H} 106 \mathrm{C} 40 \mathrm{H} 105)[41]+\delta(\mathrm{H} 107 \mathrm{C} 40 \mathrm{H} 106)[10]$ \\
\hline 1530 & 1469 & & & $\delta(\mathrm{H} 55 \mathrm{C} 5 \mathrm{H} 54)[14]+\delta(\mathrm{H} 91 \mathrm{C} 30 \mathrm{H} 90)[26]+\delta(\mathrm{H} 92 \mathrm{C} 30 \mathrm{H} 91)[11]$ \\
\hline 1530 & 1469 & & 1470 & $\delta(\mathrm{H} 70 \mathrm{C} 17 \mathrm{H} 72)[39]+\delta(\mathrm{H} 71 \mathrm{C} 17 \mathrm{H} 70)[13]$ \\
\hline 1528 & 1467 & & & $\delta(\mathrm{H} 64 \mathrm{C} 14 \mathrm{H} 66)[17]+\delta(\mathrm{H} 70 \mathrm{C} 17 \mathrm{H} 72)[11]+\delta(\mathrm{H} 72 \mathrm{C} 17 \mathrm{H} 71)[19]$ \\
\hline 1528 & 1467 & & & $\delta(\mathrm{H} 54 \mathrm{C} 5 \mathrm{H} 56)[11]+\delta(\mathrm{H} 55 \mathrm{C} 5 \mathrm{H} 54)[26]+\delta(\mathrm{H} 91 \mathrm{C} 30 \mathrm{H} 90)[19]$ \\
\hline 1526 & 1465 & & & $\begin{array}{l}\delta(\mathrm{H} 97 \mathrm{C} 36 \mathrm{H} 96)[19]+\delta(\mathrm{H} 101 \mathrm{C} 37 \mathrm{H} 100)[10]+\delta(\mathrm{H} 98 \mathrm{C} 36 \mathrm{H} 97)[24]+ \\
\delta(\mathrm{H} 100 \mathrm{C} 37 \mathrm{H} 99)[10]\end{array}$ \\
\hline 1524 & 1463 & $1460 \mathrm{vs}$ & & $\delta(\mathrm{H} 111 \mathrm{C} 45 \mathrm{H} 113)[35]+\delta(\mathrm{H} 112 \mathrm{C} 45 \mathrm{H} 111)[14]+\delta(\mathrm{H} 116 \mathrm{C} 47 \mathrm{H} 115)[15]$ \\
\hline 1524 & 1463 & & & $\delta(\mathrm{H} 63 \mathrm{O} 13 \mathrm{C} 12)[14]+\delta(\mathrm{H} 64 \mathrm{C} 14 \mathrm{H} 66)[14]+\delta(\mathrm{H} 65 \mathrm{C} 14 \mathrm{H} 64)[33]$ \\
\hline 1521 & 1460 & & & $\delta(\mathrm{H} 68 \mathrm{C} 15 \mathrm{H} 67)[12]+\delta(\mathrm{H} 74 \mathrm{C} 18 \mathrm{H} 73)[50]$ \\
\hline 1519 & 1458 & & & $\delta(\mathrm{H} 84 \mathrm{C} 26 \mathrm{H} 86)[26]+\delta(\mathrm{H} 86 \mathrm{C} 26 \mathrm{H} 85)[25]$ \\
\hline 1518 & 1457 & & & $\delta(\mathrm{H} 105 \mathrm{C} 40 \mathrm{H} 107)[14]+\delta(\mathrm{H} 120 \mathrm{C} 51 \mathrm{H} 119)[11]+\delta(\mathrm{H} 121 \mathrm{C} 51 \mathrm{H} 120)[34]$ \\
\hline 1517 & 1456 & & & $\delta(\mathrm{H} 80 \mathrm{C} 22 \mathrm{H} 79)[31]+\delta(\mathrm{H} 79 \mathrm{C} 22 \mathrm{H} 81)[26]$ \\
\hline 1516 & 1455 & & & $\delta(\mathrm{H} 105 \mathrm{C} 40 \mathrm{H} 107)[24]+\delta(\mathrm{H} 107 \mathrm{C} 40 \mathrm{H} 106)[30]+\delta(\mathrm{H} 121 \mathrm{C} 51 \mathrm{H} 120)[10] \mathrm{sci}$ \\
\hline
\end{tabular}




\begin{tabular}{|c|c|c|c|c|}
\hline 1511 & 1451 & & & $\begin{array}{l}\delta(\mathrm{H} 97 \mathrm{C} 36 \mathrm{H} 96)[17]+\delta(\mathrm{H} 101 \mathrm{C} 37 \mathrm{H} 100)[15]+\delta(\mathrm{H} 99 \mathrm{C} 37 \mathrm{H} 101)[13] \\
\delta(\mathrm{H} 103 \mathrm{C} 38 \mathrm{H} 102)[11]\end{array}$ \\
\hline 1511 & 1451 & $1451 \mathrm{vs}$ & 1452 & $\delta(\mathrm{H} 86 \mathrm{C} 26 \mathrm{H} 85)[14]+\delta(\mathrm{H} 89 \mathrm{C} 29 \mathrm{H} 88)[55]$ \\
\hline 1510 & 1450 & & & $\delta(\mathrm{H} 68 \mathrm{C} 15 \mathrm{H} 67)[48]$ \\
\hline 1510 & 1450 & & & $\delta(\mathrm{H} 112 \mathrm{C} 45 \mathrm{H} 111)[10]+\delta(\mathrm{H} 113 \mathrm{C} 45 \mathrm{H} 112)[18]+\delta(\mathrm{H} 115 \mathrm{C} 47 \mathrm{H} 114)[23]$ \\
\hline 1506 & 1446 & & & $\delta(\mathrm{H} 103 \mathrm{C} 38 \mathrm{H} 102)[60]$ \\
\hline 1502 & 1442 & & & $\delta(\mathrm{H} 97 \mathrm{C} 36 \mathrm{H} 96)[16]+\delta(\mathrm{H} 98 \mathrm{C} 36 \mathrm{H} 97)[12]+\delta(\mathrm{H} 99 \mathrm{C} 37 \mathrm{H} 101)[43]$ \\
\hline 1499 & 1439 & & & $\delta(\mathrm{H} 63 \mathrm{O} 13 \mathrm{C} 12)[48]+\delta(\mathrm{H} 65 \mathrm{C} 14 \mathrm{H} 64)[15]+\delta(\mathrm{H} 66 \mathrm{C} 14 \mathrm{H} 65)[17]$ \\
\hline 1496 & 1436 & & & $\delta(\mathrm{H} 114 \mathrm{C} 47 \mathrm{H} 116)[17]+\delta(\mathrm{H} 110 \mathrm{C} 43 \mathrm{H} 109)[64]$ \\
\hline 1480 & 1421 & & 1430 & $\delta(\mathrm{H} 75 \mathrm{C} 20 \mathrm{H} 77)[28]+\delta(\mathrm{H} 77 \mathrm{C} 20 \mathrm{H} 76)[11]+\delta(\mathrm{H} 76 \mathrm{C} 20 \mathrm{H} 75)[34]$ \\
\hline 1469 & 1410 & & & $\begin{array}{l}\delta(\mathrm{H} 97 \mathrm{C} 36 \mathrm{H} 96)[16]+\delta(\mathrm{H} 96 \mathrm{C} 36 \mathrm{H} 98)[15]+\delta(\mathrm{H} 98 \mathrm{C} 36 \mathrm{H} 97)[13] \\
\delta(\mathrm{H} 99 \mathrm{C} 37 \mathrm{H} 101)[12]+\delta(\mathrm{H} 100 \mathrm{C} 37 \mathrm{H} 99)[13]\end{array}$ \\
\hline 1466 & 1407 & & & $\delta(\mathrm{H} 78 \mathrm{C} 21 \mathrm{~N} 19)[17] \delta(\mathrm{H} 82 \mathrm{C} 23 \mathrm{O} 24)[11]$ \\
\hline 1462 & 1404 & & & $\delta(\mathrm{H} 57 \mathrm{C} 6 \mathrm{O} 7)[26]+\delta(\mathrm{H} 108 \mathrm{C} 42 \mathrm{O} 52)[27]+\tau(\mathrm{H} 108 \mathrm{C} 42 \mathrm{O} 52 \mathrm{C} 50)[16]$ \\
\hline 1458 & 1400 & & 1408 & $\delta(\mathrm{H} 83 \mathrm{O} 27 \mathrm{C} 25)[19]+\delta(\mathrm{H} 84 \mathrm{C} 26 \mathrm{H} 86)[10]+\tau(\mathrm{H} 87 \mathrm{C} 28 \mathrm{O} 1 \mathrm{C} 2)[12]$ \\
\hline 1457 & 1399 & $1403 \mathrm{~m}$ & & $\tau(\mathrm{H} 93 \mathrm{C} 31 \mathrm{O} 41 \mathrm{C} 39)[16]$ \\
\hline 1453 & 1395 & & & $\tau(\mathrm{H} 93 \mathrm{C} 31 \mathrm{O} 41 \mathrm{C} 39)[14]$ \\
\hline 1452 & 1394 & & & $\delta(\mathrm{H} 117 \mathrm{C} 48 \mathrm{O} 49)[10]+\gamma(\mathrm{C} 48 \mathrm{C} 50 \mathrm{C} 44 \mathrm{H} 117)[10]$ \\
\hline 1450 & 1392 & & & $\delta(\mathrm{H} 78 \mathrm{C} 21 \mathrm{~N} 19)[14]+\delta(\mathrm{H} 82 \mathrm{C} 23 \mathrm{O} 24)[14]+\gamma(\mathrm{C} 23 \mathrm{C} 25 \mathrm{C} 21 \mathrm{H} 82)[11]$ \\
\hline 1445 & 1387 & $1391 \mathrm{~m}$ & 1393 & $\delta(\mathrm{H} 93 \mathrm{C} 31 \mathrm{O} 41)[12]$ \\
\hline 1442 & 1384 & & & $\delta(\mathrm{N} 35 \mathrm{C} 34 \mathrm{H} 95)[20]$ \\
\hline 1441 & 1383 & & & $\delta(\mathrm{H} 90 \mathrm{C} 30 \mathrm{H} 92)[30]+\delta(\mathrm{H} 91 \mathrm{C} 30 \mathrm{H} 90)[30]+\delta(\mathrm{H} 92 \mathrm{C} 30 \mathrm{H} 91)[28]$ \\
\hline 1439 & 1381 & & & $\begin{array}{l}\delta(\mathrm{H} 70 \mathrm{C} 17 \mathrm{H} 72)[20]+\delta(\mathrm{H} 71 \mathrm{C} 17 \mathrm{H} 70)[19]+\delta(\mathrm{H} 72 \mathrm{C} 17 \mathrm{H} 71)[21]+ \\
\delta(\mathrm{H} 78 \mathrm{C} 21 \mathrm{~N} 19)[12]\end{array}$ \\
\hline 1437 & 1380 & & & $\tau(\mathrm{H} 104 \mathrm{C} 39 \mathrm{O} 41 \mathrm{C} 31)[16]$ \\
\hline 1436 & 1379 & & & $\begin{array}{l}\delta(\mathrm{H} 123 \mathrm{O} 24 \mathrm{C} 23)[13]+\delta(\mathrm{H} 80 \mathrm{C} 22 \mathrm{H} 79)[23]+\delta(\mathrm{H} 81 \mathrm{C} 22 \mathrm{H} 80)[16]+ \\
\delta(\mathrm{H} 79 \mathrm{C} 22 \mathrm{H} 81)[23]\end{array}$ \\
\hline 1436 & 1379 & & & $\delta(\mathrm{H} 64 \mathrm{C} 14 \mathrm{H} 66)[11]+\delta(\mathrm{H} 65 \mathrm{C} 14 \mathrm{H} 64)[20]+\delta(\mathrm{H} 66 \mathrm{C} 14 \mathrm{H} 65)[25]$ \\
\hline 1432 & 1375 & & & $\delta(\mathrm{H} 57 \mathrm{C} 6 \mathrm{O} 7)[10]+\delta(\mathrm{H} 120 \mathrm{C} 51 \mathrm{H} 119)[12]+\delta(\mathrm{H} 121 \mathrm{C} 51 \mathrm{H} 120)[13]$ \\
\hline 1431 & 1374 & & & $\delta(\mathrm{H} 84 \mathrm{C} 26 \mathrm{H} 86)[10]+\delta(\mathrm{H} 86 \mathrm{C} 26 \mathrm{H} 85)[14]+\tau(\mathrm{H} 87 \mathrm{C} 28 \mathrm{O} 1 \mathrm{C} 2)[11]$ \\
\hline 1430 & 1373 & & & $\delta(\mathrm{H} 57 \mathrm{C} 6 \mathrm{O} 7)[10]+\delta(\mathrm{H} 108 \mathrm{C} 42 \mathrm{O} 52)[11]+\delta(\mathrm{H} 121 \mathrm{C} 51 \mathrm{H} 120)[11]$ \\
\hline 1428 & 1371 & & & $\delta(\mathrm{H} 111 \mathrm{C} 45 \mathrm{H} 113)[24]+\delta(\mathrm{H} 112 \mathrm{C} 45 \mathrm{H} 111)[22]+\delta(\mathrm{H} 113 \mathrm{C} 45 \mathrm{H} 112)[18]$ \\
\hline 1426 & 1369 & $1371 \mathrm{~m}$ & 1377 & $\delta(\mathrm{H} 56 \mathrm{C} 5 \mathrm{H} 55)[10]$ wag. \\
\hline 1421 & 1364 & & & $\tau(\mathrm{H} 104 \mathrm{C} 39 \mathrm{O} 41 \mathrm{C} 31)[21]$ \\
\hline 1419 & 1362 & & & $\delta(\mathrm{H} 62 \mathrm{C} 10 \mathrm{O} 11)[10]$ \\
\hline 1415 & 1358 & $1364 \mathrm{~m}$ & & $\delta(\mathrm{H} 78 \mathrm{C} 21 \mathrm{~N} 19)[17] \mathrm{wag}$. \\
\hline 1413 & 1356 & & & $\begin{array}{l}\delta(\mathrm{H} 62 \mathrm{C} 10 \mathrm{O} 11)[10]+\delta(\mathrm{H} 117 \mathrm{C} 48 \mathrm{O} 49)[10]+\tau(\mathrm{H} 118 \mathrm{C} 50 \mathrm{O} 52 \mathrm{C} 42)[10]+ \\
\gamma(\mathrm{C} 48 \mathrm{C} 50 \mathrm{C} 44 \mathrm{H} 117)[10]\end{array}$ \\
\hline 1410 & 1354 & & & $\delta(\mathrm{C} 43 \mathrm{C} 42 \mathrm{H} 108)[10]+\delta(\mathrm{C} 8 \mathrm{C} 6 \mathrm{H} 57)[15]+\delta(\mathrm{C} 25 \mathrm{O} 27 \mathrm{H} 83)[10]$ \\
\hline 1410 & 1354 & & & $\delta(\mathrm{H} 95 \mathrm{C} 34 \mathrm{C} 38)[22]$ \\
\hline 1409 & 1353 & & & $\delta(\mathrm{C} 25 \mathrm{C} 23 \mathrm{H} 82)[20]+\delta(\mathrm{C} 21 \mathrm{C} 23 \mathrm{H} 82)[12]+\delta(\mathrm{C} 43 \mathrm{C} 42 \mathrm{H} 108)[22]$ \\
\hline 1405 & 1349 & $1354 \mathrm{~m}$ & & $\tau(\mathrm{H} 69 \mathrm{C} 16 \mathrm{C} 18 \mathrm{~N} 19)[26]$ \\
\hline 1399 & 1343 & & 1343 & $\delta(\mathrm{H} 123 \mathrm{O} 24 \mathrm{C} 23)[11]+\tau(\mathrm{H} 78 \mathrm{C} 21 \mathrm{~N} 19 \mathrm{C} 18)[25]+\gamma(\mathrm{O} 24 \mathrm{C} 21 \mathrm{C} 25 \mathrm{C} 23)[13]$ \\
\hline 1394 & 1338 & & & $\delta(\mathrm{H} 93 \mathrm{C} 31 \mathrm{O} 41)[12]+\delta(\mathrm{H} 104 \mathrm{C} 39 \mathrm{C} 40)[10]+\tau(\mathrm{H} 62 \mathrm{C} 10 \mathrm{O} 11 \mathrm{C} 31)[10]$ \\
\hline 1392 & 1336 & & & $\delta(\mathrm{H} 57 \mathrm{C} 6 \mathrm{O} 7)[20]+\tau(\mathrm{H} 108 \mathrm{C} 42 \mathrm{O} 52 \mathrm{C} 50)[20]+\tau(\mathrm{H} 110 \mathrm{C} 43 \mathrm{C} 44 \mathrm{C} 45)[15]$ \\
\hline 1383 & 1328 & $1334 \mathrm{~m}$ & & $\delta(\mathrm{H} 87 \mathrm{C} 28 \mathrm{O} 1)[32]$ \\
\hline
\end{tabular}




\begin{tabular}{|c|c|c|c|c|}
\hline 1381 & 1326 & & & $\delta(\mathrm{H} 118 \mathrm{C} 50 \mathrm{C} 51)[30]+\tau(\mathrm{H} 118 \mathrm{C} 50 \mathrm{O} 52 \mathrm{C} 42)[10]$ \\
\hline 1377 & 1322 & $1327 \mathrm{~m}$ & & $\tau(\mathrm{H} 108 \mathrm{C} 42 \mathrm{O} 52 \mathrm{C} 50)[10]$ \\
\hline 1376 & 1321 & & & $\delta(\mathrm{H} 118 \mathrm{C} 50 \mathrm{C} 51)[27]+\delta(\mathrm{H} 93 \mathrm{C} 31 \mathrm{O} 41)[12]$ \\
\hline 1365 & 1310 & & & $\tau(\mathrm{H} 95 \mathrm{C} 34 \mathrm{~N} 35 \mathrm{C} 36)[29]$ \\
\hline 1364 & 1309 & & & $\delta(\mathrm{H} 67 \mathrm{C} 15 \mathrm{C} 16)[12]+\delta(\mathrm{H} 69 \mathrm{C} 16 \mathrm{C} 17)[12]$ \\
\hline 1362 & 1308 & & 1317 & $\delta(\mathrm{H} 53 \mathrm{C} 4 \mathrm{C} 2)[14]+\delta(\mathrm{H} 87 \mathrm{C} 28 \mathrm{O} 1)[14]+\tau(\mathrm{H} 53 \mathrm{C} 4 \mathrm{C} 2 \mathrm{O} 1)[23]$ \\
\hline 1360 & 1306 & & & $\delta(\mathrm{H} 73 \mathrm{C} 18 \mathrm{~N} 19)[25]$ \\
\hline 1354 & 1300 & & & $\delta(\mathrm{H} 82 \mathrm{C} 23 \mathrm{O} 24)[12]+\gamma(\mathrm{C} 23 \mathrm{C} 25 \mathrm{C} 21 \mathrm{H} 82)[11]$ \\
\hline 1349 & 1295 & $1295 \mathrm{w}$ & & $\delta(\mathrm{H} 53 \mathrm{C} 4 \mathrm{C} 2)[11]+\delta(\mathrm{H} 58 \mathrm{C} 8 \mathrm{C} 9)[16]+\tau(\mathrm{H} 58 \mathrm{C} 8 \mathrm{C} 10 \mathrm{C} 12)[11]$ \\
\hline 1337 & 1284 & & & $\delta(\mathrm{H} 94 \mathrm{C} 32 \mathrm{O} 33)[16]+\gamma(\mathrm{C} 32 \mathrm{C} 34 \mathrm{C} 31 \mathrm{H} 94)[13]$ \\
\hline 1333 & 1280 & & & $\delta(\mathrm{H} 109 \mathrm{C} 43 \mathrm{C} 44)[16]+\tau(\mathrm{H} 62 \mathrm{C} 10 \mathrm{O} 11 \mathrm{C} 31)[10]$ \\
\hline 1330 & 1277 & & & $\delta(\mathrm{H} 67 \mathrm{C} 15 \mathrm{C} 16)[10]+\delta(\mathrm{H} 69 \mathrm{C} 16 \mathrm{C} 17)[17]+\delta(\mathrm{H} 109 \mathrm{C} 43 \mathrm{C} 44)[13]$ \\
\hline 1329 & 1276 & & 1283 & $\delta(\mathrm{H} 83 \mathrm{O} 27 \mathrm{C} 25)[14]+\delta(\mathrm{H} 88 \mathrm{C} 29 \mathrm{C} 30)[19]+\tau(\mathrm{H} 87 \mathrm{C} 28 \mathrm{O} 1 \mathrm{C} 2)[35]$ \\
\hline 1326 & 1273 & & & $\tau(\mathrm{H} 58 \mathrm{C} 8 \mathrm{C} 10 \mathrm{C} 12)[16]+\tau(\mathrm{H} 62 \mathrm{C} 10 \mathrm{O} 11 \mathrm{C} 31)[16]$ \\
\hline 1323 & 1270 & & & $\tau(\mathrm{H} 98 \mathrm{C} 36 \mathrm{~N} 35 \mathrm{C} 34)[14]$ \\
\hline 1319 & 1266 & $1272 \mathrm{~m}$ & 1269 & $\begin{array}{l}\delta(\mathrm{H} 117 \mathrm{C} 48 \mathrm{O} 49)[18]+\tau(\mathrm{H} 118 \mathrm{C} 50 \mathrm{O} 52 \mathrm{C} 42)[15]+\gamma(\mathrm{C} 48 \mathrm{C} 50 \mathrm{C} 44 \mathrm{H} 117)[11] \\
+\gamma(\mathrm{O} 49 \mathrm{C} 44 \mathrm{C} 50 \mathrm{C} 48)[11]\end{array}$ \\
\hline 1319 & 1266 & & & $\delta(\mathrm{H} 88 \mathrm{C} 29 \mathrm{C} 30)[23]+\tau(\mathrm{H} 88 \mathrm{C} 29 \mathrm{C} 28 \mathrm{C} 25)[12]+\tau(\mathrm{H} 89 \mathrm{C} 29 \mathrm{C} 28 \mathrm{C} 25)[26]+$ \\
\hline 1313 & 1260 & & & $\delta(\mathrm{H} 58 \mathrm{C} 8 \mathrm{C} 9)[13]+\tau(\mathrm{H} 57 \mathrm{C} 6 \mathrm{O} 7 \mathrm{C} 42)[10]$ \\
\hline 1311 & 1259 & & & $\delta(\mathrm{H} 123 \mathrm{O} 24 \mathrm{C} 23)[20]+\delta(\mathrm{H} 122 \mathrm{O} 33 \mathrm{C} 32)[15]+\tau(\mathrm{H} 78 \mathrm{C} 21 \mathrm{~N} 19 \mathrm{C} 18)[13]$ \\
\hline 1310 & 1258 & & & $\delta(\mathrm{H} 122 \mathrm{O} 33 \mathrm{C} 32)[12]+\delta(\mathrm{H} 58 \mathrm{C} 8 \mathrm{C} 9)[12]+\tau(\mathrm{H} 57 \mathrm{C} 6 \mathrm{O} 7 \mathrm{C} 42)[10]$ \\
\hline 1301 & 1249 & $1251 \mathrm{w}$ & 1250 & $\delta(\mathrm{H} 67 \mathrm{C} 15 \mathrm{C} 16)[12]+\tau(\mathrm{H} 68 \mathrm{C} 15 \mathrm{C} 16 \mathrm{C} 17)[12]+\tau(\mathrm{H} 69 \mathrm{C} 16 \mathrm{C} 18 \mathrm{~N} 19)[12]$ \\
\hline 1297 & 1245 & $1232 \mathrm{~m}$ & & $\tau(\mathrm{H} 103 \mathrm{C} 38 \mathrm{C} 39 \mathrm{C} 40)[22]$ \\
\hline 1271 & 1220 & $1225 \mathrm{~m}$ & & $\delta(\mathrm{H} 124 \mathrm{O} 49 \mathrm{C} 48)[34]$ \\
\hline 1250 & 1200 & & & $\tau(\mathrm{H} 74 \mathrm{C} 18 \mathrm{~N} 19 \mathrm{C} 20)[11]+\tau(\mathrm{H} 76 \mathrm{C} 20 \mathrm{~N} 19 \mathrm{C} 18)[19]$ \\
\hline 1239 & 1189 & & & $\tau(\mathrm{H} 115 \mathrm{C} 47 \mathrm{O} 46 \mathrm{C} 44)[16]+\tau(\mathrm{H} 116 \mathrm{C} 47 \mathrm{O} 46 \mathrm{C} 44)[16]$ \\
\hline 1235 & 1186 & $1187 \mathrm{~m}$ & 1188 & $\delta(\mathrm{H} 95 \mathrm{C} 34 \mathrm{C} 38)[10]+\delta(\mathrm{H} 102 \mathrm{C} 38 \mathrm{C} 39)[19]$ \\
\hline 1232 & 1183 & & & $\tau(\mathrm{H} 85 \mathrm{C} 26 \mathrm{C} 25 \mathrm{C} 23)[10]$ \\
\hline 1224 & 1175 & & & $v(\mathrm{~N} 19 \mathrm{C} 20)[20]+\delta(\mathrm{C} 18 \mathrm{~N} 19 \mathrm{C} 21)[16]$ \\
\hline 1218 & 1169 & & & $\delta(\mathrm{C} 34 \mathrm{C} 32 \mathrm{H} 94)$ \\
\hline 1216 & 1167 & & & $\tau(\mathrm{H} 110 \mathrm{C} 43 \mathrm{C} 44 \mathrm{C} 45)[10]$ \\
\hline 1210 & 1162 & & 1166 & $v(\mathrm{O} 1 \mathrm{C} 2)[36]+\delta(\mathrm{H} 53 \mathrm{C} 4 \mathrm{C} 2)[14]$ \\
\hline 1201 & 1153 & & & $\tau(\mathrm{H} 97 \mathrm{C} 36 \mathrm{~N} 35 \mathrm{C} 34)[18]+\tau(\mathrm{H} 101 \mathrm{C} 37 \mathrm{~N} 35 \mathrm{C} 34)[21]$ \\
\hline 1198 & 1150 & $1154 \mathrm{~m}$ & 1156 & $\tau(\mathrm{H} 66 \mathrm{C} 14 \mathrm{C} 12 \mathrm{C} 10)[13]$ \\
\hline 1194 & 1146 & & & $v(\mathrm{C} 25 \mathrm{O} 27)+v(\mathrm{C} 25 \mathrm{C} 28)+\delta(\mathrm{C} 30 \mathrm{C} 29 \mathrm{C} 28)$ \\
\hline 1193 & 1145 & & & $v(\mathrm{C} 12 \mathrm{C} 10)+\delta(\mathrm{C} 14 \mathrm{C} 12 \mathrm{C} 10)$ \\
\hline 1191 & 1143 & & & $\begin{array}{l}\delta(\mathrm{H} 114 \mathrm{C} 47 \mathrm{H} 116)[13]+\delta(\mathrm{H} 115 \mathrm{C} 47 \mathrm{H} 114)[13]+\tau(\mathrm{H} 114 \mathrm{C} 47 \mathrm{O} 46 \mathrm{C} 44)[28] \\
+\tau(\mathrm{H} 115 \mathrm{C} 47 \mathrm{O} 46 \mathrm{C} 44)[19]+\tau(\mathrm{H} 116 \mathrm{C} 47 \mathrm{O} 46 \mathrm{C} 44)[20]\end{array}$ \\
\hline 1189 & 1141 & & 1145 & $\delta(\mathrm{C} 25 \mathrm{O} 27 \mathrm{H} 83)+\delta(\mathrm{N} 19 \mathrm{C} 20 \mathrm{H} 76)+\mathrm{v}(\mathrm{C} 25 \mathrm{C} 28)$ \\
\hline 1189 & 1141 & & & $v(C 45 C 44)[10]$ \\
\hline 1186 & 1139 & & & $\tau(\mathrm{H} 106 \mathrm{C} 40 \mathrm{C} 39 \mathrm{C} 38)[11]$ \\
\hline 1182 & 1135 & & & $\delta(\mathrm{C} 48 \mathrm{O} 49 \mathrm{H} 124)+v(\mathrm{C} 42 \mathrm{O} 7)+v(\mathrm{O} 46 \mathrm{C} 47)$ \\
\hline 1172 & 1125 & & & $\tau(\mathrm{H} 75 \mathrm{C} 20 \mathrm{~N} 19 \mathrm{C} 18)[10]+\tau(\mathrm{H} 77 \mathrm{C} 20 \mathrm{~N} 19 \mathrm{C} 18)[21]$ \\
\hline 1170 & 1123 & & & $\delta(\mathrm{C} 34 \mathrm{C} 38 \mathrm{C} 39)+v(\mathrm{C} 32 \mathrm{C} 31)$ \\
\hline 1169 & 1122 & & 1123 & $v(\mathrm{C} 50 \mathrm{C} 51)+v(\mathrm{C} 50 \mathrm{C} 48)$ \\
\hline 1169 & 1122 & & & $v(\mathrm{C} 39 \mathrm{O} 41)+v(\mathrm{C} 48 \mathrm{C} 50)$ \\
\hline
\end{tabular}




\begin{tabular}{|c|c|c|c|c|}
\hline 1167 & 1120 & $1121 \mathrm{~m}$ & & $v(\mathrm{C} 9 \mathrm{C} 8)[12]+v(\mathrm{C} 6 \mathrm{C} 8)$ \\
\hline 1158 & 1112 & & & $v(\mathrm{C} 15 \mathrm{C} 16)+v(\mathrm{C} 16 \mathrm{C} 18)$ \\
\hline 1153 & 1107 & & 1106 & $v(\mathrm{O} 52 \mathrm{C} 42)[31]+v(\mathrm{C} 43 \mathrm{C} 42)[19]$ \\
\hline 1143 & 1097 & & & $v(\mathrm{C} 31 \mathrm{O} 11)+v(\mathrm{C} 16 \mathrm{C} 15)$ \\
\hline 1140 & 1094 & & & $v(\mathrm{C} 32 \mathrm{O} 33)+\delta(\mathrm{C} 31 \mathrm{C} 32 \mathrm{C} 34)$ \\
\hline 1139 & 1093 & $1096 \mathrm{~m}$ & & $v(C 4 C 6)+v(C 4 C 5)+v(C 9 C 8)$ \\
\hline 1136 & 1091 & & 1096 & $\delta(\mathrm{N} 35 \mathrm{C} 36 \mathrm{H} 98)+\tau(\mathrm{C} 34 \mathrm{~N} 35 \mathrm{C} 36 \mathrm{H} 97)$ \\
\hline 1135 & 1090 & & & $\tau(\mathrm{H} 91 \mathrm{C} 30 \mathrm{C} 29 \mathrm{C} 28)[10]$ \\
\hline 1130 & 1085 & & & $\tau(\mathrm{H} 97 \mathrm{C} 36 \mathrm{~N} 35 \mathrm{C} 34)[16]+\tau(\mathrm{H} 101 \mathrm{C} 37 \mathrm{~N} 35 \mathrm{C} 34)[11]$ \\
\hline 1126 & 1081 & & & $v(\mathrm{C} 4 \mathrm{C} 6)[13]+\tau(\mathrm{H} 56 \mathrm{C} 5 \mathrm{C} 4 \mathrm{C} 6)[15]$ \\
\hline 1122 & 1077 & & & $v(\mathrm{O} 46 \mathrm{C} 47)[40]$ \\
\hline 1116 & 1071 & & 1083 & $v(\mathrm{O} 11 \mathrm{C} 31)[14]$ \\
\hline 1109 & 1065 & & & $v(\mathrm{O} 49 \mathrm{C} 48)[21]$ \\
\hline 1107 & 1063 & & & $v(\mathrm{C} 22 \mathrm{C} 21)+v(\mathrm{C} 21 \mathrm{~N} 19)$ \\
\hline 1104 & 1060 & $1062 \mathrm{~m}$ & & $v(\mathrm{O} 46 \mathrm{C} 47)[10]+v(\mathrm{O} 49 \mathrm{C} 48)[16]$ \\
\hline 1094 & 1050 & & & $v(\mathrm{C} 29 \mathrm{C} 28)[18]$ \\
\hline 1092 & 1048 & & & $v(\mathrm{~N} 35 \mathrm{C} 36)[11]+v(\mathrm{O} 33 \mathrm{C} 32)[21]+v(\mathrm{~N} 35 \mathrm{C} 34)[10]$ \\
\hline 1088 & 1044 & & 1049 & $v(\mathrm{O} 49 \mathrm{C} 48)[10]+v(\mathrm{O} 52 \mathrm{C} 50)[12]+\tau(\mathrm{H} 121 \mathrm{C} 51 \mathrm{C} 50 \mathrm{C} 48)[10]$ \\
\hline 1087 & 1044 & & & $v(\mathrm{C} 21 \mathrm{~N} 19) v(\mathrm{C} 21 \mathrm{C} 23) \delta(\mathrm{C} 18 \mathrm{C} 16 \mathrm{C} 15)$ \\
\hline 1083 & 1040 & & & $v(\mathrm{~N} 35 \mathrm{C} 36)[10]+v(\mathrm{~N} 35 \mathrm{C} 37)[14]+\tau(\mathrm{H} 98 \mathrm{C} 36 \mathrm{~N} 35 \mathrm{C} 34)[10]$ \\
\hline 1081 & 1038 & $1042 \mathrm{~m}$ & & $v(\mathrm{C} 16 \mathrm{C} 17)+v(\mathrm{C} 16 \mathrm{C} 15)+v(\mathrm{C} 4 \mathrm{C} 5)$ \\
\hline 1077 & 1034 & & & $v(\mathrm{C} 5 \mathrm{C} 4)[10]$ \\
\hline 1075 & 1032 & & 1032 & $v(\mathrm{O} 41 \mathrm{C} 31)[20]$ \\
\hline 1071 & 1028 & & & $v(\mathrm{C} 4 \mathrm{C} 6)+v(\mathrm{C} 4 \mathrm{C} 5)+v(\mathrm{C} 9 \mathrm{C} 8)$ \\
\hline 1070 & 1027 & & & $v(\mathrm{~N} 19 \mathrm{C} 18)[13]$ \\
\hline 1065 & 1022 & & & $v(\mathrm{C} 32 \mathrm{C} 31)+v(\mathrm{C} 34 \mathrm{C} 32)$ \\
\hline 1059 & 1017 & & 1018 & $v(\mathrm{C} 30 \mathrm{C} 29)[27]$ \\
\hline 1055 & 1013 & & & $v(\mathrm{C} 34 \mathrm{C} 38)+v(\mathrm{C} 34 \mathrm{C} 32)+v(\mathrm{C} 8 \mathrm{C} 10)$ \\
\hline 1045 & 1003 & $1000 \mathrm{w}$ & 1004 & $v(\mathrm{~N} 35 \mathrm{C} 34)[12]+v(\mathrm{C} 40 \mathrm{C} 39)[15]$ \\
\hline 1031 & 990 & $988 \mathrm{w}$ & 992 & $v(\mathrm{O} 7 \mathrm{C} 42)[18]+v(\mathrm{O} 11 \mathrm{C} 10)[10]$ \\
\hline 1022 & 981 & & 978 & $v(\mathrm{O} 11 \mathrm{C} 10)[14]$ \\
\hline 1019 & 978 & $980 \mathrm{w}$ & 977 & $v(\mathrm{O} 24 \mathrm{C} 23)[10]$ \\
\hline 1013 & 972 & & 966 & $v(\mathrm{O} 7 \mathrm{C} 6)[13]+v(\mathrm{C} 43 \mathrm{C} 42)[15]$ \\
\hline 1001 & 961 & $963 \mathrm{~m}$ & 956 & $v(\mathrm{O} 7 \mathrm{C} 6)[12]$ \\
\hline 998 & 958 & & & $v(\mathrm{C} 12 \mathrm{C} 15)[14]+\tau(\mathrm{H} 64 \mathrm{C} 14 \mathrm{C} 12 \mathrm{C} 10)[15]$ \\
\hline 989 & 949 & & & $v(\mathrm{O} 24 \mathrm{C} 23)[20]+\tau(\mathrm{H} 78 \mathrm{C} 21 \mathrm{~N} 19 \mathrm{C} 18)[10]$ \\
\hline 985 & 946 & & & $\delta(\mathrm{H} 102 \mathrm{C} 38 \mathrm{C} 39)[13]$ \\
\hline 977 & 938 & & 943 & $v(\mathrm{C} 30 \mathrm{C} 29)[27]$ \\
\hline 975 & 936 & & & $v(\mathrm{O} 13 \mathrm{C} 12)[15]$ \\
\hline 966 & 927 & $930 \mathrm{~m}$ & 931 & $v(\mathrm{C} 23 \mathrm{C} 25)[11]+\tau(\mathrm{H} 85 \mathrm{C} 26 \mathrm{C} 25 \mathrm{C} 23)[14]$ \\
\hline 964 & 925 & & & $v($ C 50 C 51$)+v($ C 50 C 48$)+v($ C43C44) \\
\hline 963 & 924 & & & $v(\mathrm{C} 18 \mathrm{C} 16)+v(\mathrm{~N} 19 \mathrm{C} 21)$ \\
\hline 957 & 919 & & & $v(\mathrm{C} 18 \mathrm{C} 16)+v(\mathrm{C} 16 \mathrm{C} 17)$ \\
\hline 951 & 913 & & 910 & $v(\mathrm{C} 39 \mathrm{C} 38)[10]+v(\mathrm{O} 41 \mathrm{C} 39)[12]+\tau(\mathrm{H} 106 \mathrm{C} 40 \mathrm{C} 39 \mathrm{C} 38)[23]$ \\
\hline 943 & 905 & $908 \mathrm{~m}$ & 903 & $v(\mathrm{C} 21 \mathrm{~N} 19)+\tau(\mathrm{C} 21 \mathrm{~N} 19 \mathrm{C} 18 \mathrm{H} 74)+v(\mathrm{C} 25 \mathrm{O} 27)$ \\
\hline 936 & 899 & & & $v(\mathrm{C} 6 \mathrm{C} 8)[12]$ \\
\hline
\end{tabular}




\begin{tabular}{|c|c|c|c|c|}
\hline 928 & 891 & & & $v(\mathrm{C} 28 \mathrm{O} 1)$ \\
\hline 919 & 882 & & & $v(\mathrm{C} 48 \mathrm{C} 50)$ \\
\hline 915 & 878 & $881 \mathrm{~m}$ & 898 & $v(\mathrm{O} 46 \mathrm{C} 44)[14]$ \\
\hline 909 & 873 & $874 \mathrm{~m}$ & 880 & $\delta(\mathrm{C} 28 \mathrm{O} 1 \mathrm{C} 2)$ \\
\hline 903 & 867 & $868 \mathrm{~m}$ & 866 & $\tau(\mathrm{H} 103 \mathrm{C} 38 \mathrm{C} 39 \mathrm{C} 40)[10]+\tau(\mathrm{C} 38 \mathrm{C} 39 \mathrm{C} 40 \mathrm{H} 105)$ \\
\hline 893 & 857 & & & $v(\mathrm{C} 5 \mathrm{C} 4)+v(\mathrm{C} 4 \mathrm{C} 6)$ \\
\hline 882 & 847 & & & $v(\mathrm{C} 15 \mathrm{C} 16)[10]+\tau(\mathrm{H} 67 \mathrm{C} 15 \mathrm{C} 16 \mathrm{C} 17)[13]$ \\
\hline 879 & 844 & & 848 & $\tau(\mathrm{H} 102 \mathrm{C} 38 \mathrm{C} 39 \mathrm{C} 40)[17]$ \\
\hline 876 & 841 & & & $v(\mathrm{C} 5 \mathrm{C} 4)+v(\mathrm{C} 4 \mathrm{C} 6)$ \\
\hline 872 & 837 & & & $v(C 9 C 8)[22]$ \\
\hline 861 & 827 & $834 \mathrm{~m}$ & 834 & $\delta(\mathrm{C} 43 \mathrm{C} 42 \mathrm{O} 52)$ \\
\hline 854 & 820 & & & $v(\mathrm{O} 52 \mathrm{C} 50)[14]$ \\
\hline 847 & 813 & & & $v(\mathrm{~N} 35 \mathrm{C} 36)[25]+v(\mathrm{~N} 35 \mathrm{C} 37)[16]+v(\mathrm{~N} 35 \mathrm{C} 34)[17]$ \\
\hline 845 & 811 & $811 \mathrm{~m}$ & 813 & $v(\mathrm{C} 44 \mathrm{C} 48)[12]$ \\
\hline 830 & 797 & & 805 & $v(\mathrm{O} 27 \mathrm{C} 25)[11]+v(\mathrm{C} 22 \mathrm{C} 21)[16]+\tau(\mathrm{H} 63 \mathrm{O} 13 \mathrm{C} 12 \mathrm{C} 10)[23]$ \\
\hline 812 & 780 & & 796 & $\tau(\mathrm{C} 15 \mathrm{C} 12 \mathrm{O} 13 \mathrm{H} 63)$ \\
\hline 808 & 776 & & & $\tau(\mathrm{H} 63 \mathrm{O} 13 \mathrm{C} 12 \mathrm{C} 10)[27]$ \\
\hline 803 & 771 & $774 \mathrm{~m}$ & 776 & $v(\mathrm{~N} 19 \mathrm{C} 21)[18]+\tau(\mathrm{H} 63 \mathrm{O} 13 \mathrm{C} 12 \mathrm{C} 10)[30]$ \\
\hline 785 & 754 & & 756 & $\delta(\mathrm{H} 88 \mathrm{C} 29 \mathrm{C} 30)[13]+\tau(\mathrm{H} 88 \mathrm{C} 29 \mathrm{C} 28 \mathrm{C} 25)[23]+\tau(\mathrm{H} 90 \mathrm{C} 30 \mathrm{C} 29 \mathrm{C} 28)[15]$ \\
\hline 775 & 744 & & 745 & $v(\mathrm{C} 14 \mathrm{C} 12)[17]+v(\mathrm{C} 12 \mathrm{C} 15)[17]+\delta(\mathrm{C} 12 \mathrm{C} 15 \mathrm{C} 16)[11]$ \\
\hline 764 & 733 & $731 \mathrm{~m}$ & 731 & $\gamma(\mathrm{O} 3 \mathrm{C} 4 \mathrm{O} 1 \mathrm{C} 2)[55]$ \\
\hline 741 & 711 & & 722 & $v(\mathrm{O} 46 \mathrm{C} 44)[30]+v(\mathrm{C} 45 \mathrm{C} 44)[10]$ \\
\hline 727 & 698 & $696 \mathrm{vw}$ & 697 & $\tau(\mathrm{C} 25 \mathrm{C} 23 \mathrm{O} 24 \mathrm{H} 123)+\delta(\mathrm{C} 26 \mathrm{C} 25 \mathrm{C} 28)$ \\
\hline 705 & 677 & $667 \mathrm{vw}$ & 674 & $\delta(\mathrm{O} 3 \mathrm{C} 2 \mathrm{O} 1)[19]$ \\
\hline 679 & 652 & & 663 & $v(\mathrm{C} 21 \mathrm{C} 22)+\delta(\mathrm{C} 21 \mathrm{~N} 19 \mathrm{C} 20)$ \\
\hline 668 & 641 & & 639 & $\tau(\mathrm{H} 83 \mathrm{O} 27 \mathrm{C} 25 \mathrm{C} 23)+\tau(\mathrm{H} 123 \mathrm{O} 24 \mathrm{C} 23 \mathrm{C} 21)$ \\
\hline 656 & 630 & & 629 & $\tau(\mathrm{H} 123 \mathrm{O} 24 \mathrm{C} 23 \mathrm{C} 21)[49]+\tau(\mathrm{H} 83 \mathrm{O} 27 \mathrm{C} 25 \mathrm{C} 23)[29]$ \\
\hline 640 & 614 & $612 \mathrm{~m}$ & 611 & $\delta(\mathrm{C} 38 \mathrm{C} 39 \mathrm{O} 41)+\tau(\mathrm{C} 25 \mathrm{C} 23 \mathrm{O} 24 \mathrm{H} 123)+\delta(\mathrm{C} 32 \mathrm{C} 31 \mathrm{O} 11)$ \\
\hline 631 & 606 & & & $\delta(\mathrm{C} 033 \mathrm{C} 32 \mathrm{C} 31)+\tau(\mathrm{C} 32 \mathrm{C} 34 \mathrm{C} 38 \mathrm{H} 102)$ \\
\hline 620 & 595 & & 601 & $\delta(\mathrm{O} 52 \mathrm{C} 42 \mathrm{O} 7)[20]+\delta(\mathrm{C} 50 \mathrm{O} 52 \mathrm{C} 42)[10]$ \\
\hline 613 & 588 & & 591 & $\delta(\mathrm{C} 10 \mathrm{C} 8 \mathrm{C} 6)$ \\
\hline 608 & 584 & $584 \mathrm{vw}$ & 581 & $v(\mathrm{C} 45 \mathrm{C} 44)[12]$ \\
\hline 582 & 559 & $565 \mathrm{vw}$ & 571 & $\tau(\mathrm{C} 25 \mathrm{C} 23 \mathrm{O} 24 \mathrm{H} 123)+\gamma(\mathrm{N} 19 \mathrm{C} 18 \mathrm{C} 21 \mathrm{C} 20)$ \\
\hline 575 & 552 & $561 \mathrm{vw}$ & 561 & $\delta(\mathrm{C} 22 \mathrm{C} 21 \mathrm{~N} 19)+\tau(\mathrm{C} 25 \mathrm{C} 23 \mathrm{O} 24 \mathrm{H} 123)$ \\
\hline 570 & 547 & $551 \mathrm{vw}$ & & $\delta(\mathrm{O} 52 \mathrm{C} 42 \mathrm{O} 7)[11]$ \\
\hline 549 & 527 & $537 \mathrm{w}$ & 540 & $\tau(\mathrm{H} 123 \mathrm{O} 24 \mathrm{C} 23 \mathrm{C} 21)[34]+\tau(\mathrm{H} 83 \mathrm{O} 27 \mathrm{C} 25 \mathrm{C} 23)[49]$ \\
\hline 546 & 524 & $523 \mathrm{w}$ & 524 & $\delta(\mathrm{C} 40 \mathrm{C} 39 \mathrm{O} 41)[12]$ \\
\hline 541 & 519 & & 517 & $\delta(\mathrm{C} 39 \mathrm{O} 41 \mathrm{C} 31)+\tau(\mathrm{C} 32 \mathrm{C} 34 \mathrm{C} 38 \mathrm{H} 102)$ \\
\hline 530 & 509 & & & $v(\mathrm{C} 26 \mathrm{C} 25)[11]$ \\
\hline 523 & 502 & & 506 & $\delta(\mathrm{C} 44 \mathrm{C} 48 \mathrm{C} 50)+\delta(\mathrm{C} 48 \mathrm{C} 44 \mathrm{C} 43)$ breathing \\
\hline 506 & 486 & $497 \mathrm{~m}$ & 494 & $\delta(\mathrm{O} 13 \mathrm{C} 12 \mathrm{C} 14)[10]$ \\
\hline 502 & 482 & & 487 & $\delta(\mathrm{C} 44 \mathrm{C} 48 \mathrm{C} 50)+\delta(\mathrm{C} 48 \mathrm{C} 50 \mathrm{C} 51)$ \\
\hline 493 & 473 & & & $\delta(\mathrm{C} 50 \mathrm{O} 52 \mathrm{C} 42)[10]$ \\
\hline 489 & 469 & $468 \mathrm{~m}$ & 460 & $\gamma(\mathrm{C} 39 \mathrm{C} 39 \mathrm{O} 41 \mathrm{C} 40)+\delta(\mathrm{C} 45 \mathrm{C} 44 \mathrm{C} 48)$ breathing \\
\hline 477 & 458 & & & $\delta(\mathrm{C} 37 \mathrm{~N} 35 \mathrm{C} 36)[18]$ \\
\hline 468 & 449 & & & $\delta(\mathrm{C} 48 \mathrm{C} 50 \mathrm{O} 52)+\delta(\mathrm{C} 50 \mathrm{C} 48 \mathrm{C} 44)$ \\
\hline
\end{tabular}




\begin{tabular}{|c|c|c|c|c|}
\hline 466 & 447 & & & $\tau(\mathrm{H} 122 \mathrm{O} 33 \mathrm{C} 32 \mathrm{C} 31)[38]$ \\
\hline 461 & 443 & & & $\tau(\mathrm{H} 122 \mathrm{O} 33 \mathrm{C} 32 \mathrm{C} 31)[23]$ \\
\hline 460 & 442 & $440 \mathrm{w}$ & 437 & $\tau(\mathrm{H} 122 \mathrm{O} 33 \mathrm{C} 32 \mathrm{C} 31)[10]$ \\
\hline 442 & 424 & $426 \mathrm{w}$ & & $\delta(\mathrm{C} 39 \mathrm{O} 41 \mathrm{C} 31)+\tau(\mathrm{C} 8 \mathrm{C} 10 \mathrm{C} 12 \mathrm{O} 13)$ \\
\hline 436 & 419 & & 418 & $\delta(\mathrm{C} 15 \mathrm{C} 16 \mathrm{C} 18)[18]$ \\
\hline 422 & 405 & & & $\delta(\mathrm{C} 36 \mathrm{~N} 35 \mathrm{C} 34)[12]$ \\
\hline 421 & 404 & & 408 & $\gamma(\mathrm{C} 51 \mathrm{C} 48 \mathrm{O} 52 \mathrm{C} 50)[10]$ \\
\hline 406 & 390 & $399 \mathrm{~m}$ & & $\delta(\mathrm{C} 18 \mathrm{~N} 19 \mathrm{C} 21)[11]+\delta(\mathrm{C} 14 \mathrm{C} 12 \mathrm{C} 15)[11]$ \\
\hline 400 & 384 & & & $\delta(\mathrm{C} 34 \mathrm{C} 32 \mathrm{C} 31)+\delta(\mathrm{C} 38 \mathrm{C} 39 \mathrm{O} 41)$ \\
\hline 392 & 376 & & & $\delta(\mathrm{C} 43 \mathrm{C} 42 \mathrm{O} 52)[11]$ \\
\hline 389 & 373 & $374 w$ & & $\delta(\mathrm{C} 20 \mathrm{~N} 19 \mathrm{C} 18)+\delta(\mathrm{C} 26 \mathrm{C} 25 \mathrm{C} 23)+\gamma(\mathrm{C} 36 \mathrm{C} 37 \mathrm{C} 34 \mathrm{~N} 35)$ \\
\hline 382 & 367 & & & $\delta(\mathrm{C} 47 \mathrm{O} 46 \mathrm{C} 44)+\delta(\mathrm{C} 34 \mathrm{~N} 35 \mathrm{C} 37)$ \\
\hline 375 & 360 & & & $\delta(\mathrm{C} 47 \mathrm{O} 46 \mathrm{C} 44)[16]$ \\
\hline 366 & 351 & $351 \mathrm{~m}$ & & $\delta(\mathrm{C} 36 \mathrm{~N} 35 \mathrm{C} 37)+\gamma(\mathrm{N} 19 \mathrm{C} 18 \mathrm{C} 16 \mathrm{C} 17)$ \\
\hline 359 & 345 & & & $\delta(\mathrm{C} 47 \mathrm{O} 46 \mathrm{C} 44)[13]+\gamma(\mathrm{C} 37 \mathrm{C} 34 \mathrm{C} 36 \mathrm{~N} 35)[13]$ \\
\hline 355 & 341 & & & $\delta(\mathrm{C} 47 \mathrm{O} 46 \mathrm{C} 44)+\delta(\mathrm{C} 34 \mathrm{~N} 35 \mathrm{C} 37)$ \\
\hline 348 & 334 & & & $\gamma(\mathrm{C} 37 \mathrm{C} 34 \mathrm{C} 36 \mathrm{~N} 35)[10]+\gamma(\mathrm{C} 14 \mathrm{C} 10 \mathrm{C} 15 \mathrm{C} 12)[10]$ \\
\hline 346 & 332 & & & $\delta(\mathrm{C} 45 \mathrm{C} 44 \mathrm{O} 46)[16]$ \\
\hline 343 & 329 & & & $\tau(\mathrm{C} 23 \mathrm{C} 25 \mathrm{C} 26 \mathrm{H} 85)$ \\
\hline 342 & 328 & $324 \mathrm{~m}$ & & $\delta(\mathrm{C} 20 \mathrm{~N} 19 \mathrm{C} 21)[20]+\gamma(\mathrm{C} 22 \mathrm{C} 23 \mathrm{~N} 19 \mathrm{C} 21)[10]$ \\
\hline 331 & 318 & & & $\delta(\mathrm{C} 28 \mathrm{C} 29 \mathrm{C} 30)+\delta(\mathrm{C} 21 \mathrm{~N} 19 \mathrm{C} 20)$ \\
\hline 329 & 316 & & & $\delta(\mathrm{C} 32 \mathrm{C} 34 \mathrm{C} 38)+\delta(\mathrm{C} 31 \mathrm{O} 41 \mathrm{C} 39)$ \\
\hline 320 & 307 & & & $\delta(\mathrm{N} 19 \mathrm{C} 18 \mathrm{C} 16)+\delta(\mathrm{C} 16 \mathrm{C} 15 \mathrm{C} 12)+\delta(\mathrm{C} 37 \mathrm{~N} 35 \mathrm{C} 34)$ \\
\hline 315 & 302 & & & $\tau(\mathrm{C} 10 \mathrm{C} 12 \mathrm{C} 14 \mathrm{H} 64)+\tau(\mathrm{C} 50 \mathrm{C} 48 \mathrm{O} 49 \mathrm{H} 124)$ \\
\hline 313 & 300 & & & $\delta(\mathrm{O} 49 \mathrm{C} 48 \mathrm{C} 50)[10]$ \\
\hline 310 & 298 & $299 \mathrm{~m}$ & & $\gamma(\mathrm{O} 27 \mathrm{C} 23 \mathrm{C} 28 \mathrm{C} 25)[14]+\tau(\mathrm{C} 44 \mathrm{C} 48 \mathrm{C} 49 \mathrm{H} 124)$ \\
\hline 306 & 294 & & & $\tau(\mathrm{H} 124 \mathrm{O} 49 \mathrm{C} 48 \mathrm{C} 44)[29]$ \\
\hline 303 & 291 & & & $\delta(\mathrm{C} 5 \mathrm{C} 4 \mathrm{C} 2)[10]$ \\
\hline 297 & 285 & & & $\tau(\mathrm{H} 54 \mathrm{C} 5 \mathrm{C} 4 \mathrm{C} 6)[13]+\tau(\mathrm{H} 55 \mathrm{C} 5 \mathrm{C} 4 \mathrm{C} 6)[15]+\tau(\mathrm{H} 56 \mathrm{C} 5 \mathrm{C} 4 \mathrm{C} 6)[20]$ \\
\hline 295 & 283 & & & $\tau(\mathrm{H} 59 \mathrm{C} 9 \mathrm{C} 8 \mathrm{C} 6)[16]+\tau(\mathrm{H} 60 \mathrm{C} 9 \mathrm{C} 8 \mathrm{C} 6)[12]+\tau(\mathrm{H} 61 \mathrm{C} 9 \mathrm{C} 8 \mathrm{C} 6)[12]$ \\
\hline 294 & 282 & & & $\tau(\mathrm{C} 10 \mathrm{C} 8 \mathrm{C} 9 \mathrm{H} 59)+\tau(\mathrm{C} 28 \mathrm{C} 29 \mathrm{C} 30 \mathrm{H} 90)$ \\
\hline 290 & 278 & $279 \mathrm{~m}$ & & $\tau(\mathrm{C} 6 \mathrm{C} 4 \mathrm{C} 5 \mathrm{H} 56)+\tau(\mathrm{C} 21 \mathrm{~N} 19 \mathrm{C} 20 \mathrm{H} 75)$ \\
\hline 287 & 276 & & & $\delta(\mathrm{C} 36 \mathrm{~N} 35 \mathrm{C} 34)[12]$ \\
\hline 281 & 270 & & & $\tau(\mathrm{C} 50 \mathrm{C} 48 \mathrm{O} 49 \mathrm{H} 124)+\tau(\mathrm{C} 48 \mathrm{C} 44 \mathrm{C} 45 \mathrm{H} 111)$ \\
\hline 276 & 265 & & & $\tau(\mathrm{H} 97 \mathrm{C} 36 \mathrm{~N} 35 \mathrm{C} 34)[14]+\tau(\mathrm{H} 96 \mathrm{C} 36 \mathrm{~N} 35 \mathrm{C} 34)[13]$ \\
\hline 274 & 263 & & & $\tau(\mathrm{C} 18 \mathrm{~N} 19 \mathrm{C} 20 \mathrm{H} 75)$ \\
\hline 270 & 259 & $260 \mathrm{~m}$ & & $\tau(\mathrm{C} 50 \mathrm{C} 48 \mathrm{O} 49 \mathrm{H} 124)+\tau(\mathrm{C} 18 \mathrm{~N} 19 \mathrm{C} 20 \mathrm{H} 75)+\tau(\mathrm{C} 23 \mathrm{C} 21 \mathrm{C} 22 \mathrm{H} 80)$ \\
\hline 266 & 255 & & & $\tau(\mathrm{H} 80 \mathrm{C} 22 \mathrm{C} 21 \mathrm{C} 23)[12]+\tau(\mathrm{H} 79 \mathrm{C} 22 \mathrm{C} 21 \mathrm{C} 23)[16]$ \\
\hline 265 & 254 & & & $\gamma(\mathrm{C} 45 \mathrm{C} 43 \mathrm{O} 46 \mathrm{C} 44)[14]$ \\
\hline 263 & 252 & & & $\delta(\mathrm{C} 40 \mathrm{C} 39 \mathrm{O} 41)[12]$ \\
\hline 255 & 245 & & & $\delta(\mathrm{C} 51 \mathrm{C} 50 \mathrm{O} 52)[14]+\tau(\mathrm{H} 124 \mathrm{O} 49 \mathrm{C} 48 \mathrm{C} 44)[11]$ \\
\hline 252 & 242 & & & $\delta(\mathrm{C} 22 \mathrm{C} 21 \mathrm{C} 23)[18]$ \\
\hline 250 & 240 & & & $\tau(\mathrm{C} 10 \mathrm{C} 12 \mathrm{C} 14 \mathrm{H} 64)+\tau(\mathrm{C} 50 \mathrm{C} 48 \mathrm{O} 49 \mathrm{H} 124)$ \\
\hline 246 & 236 & & & $\begin{array}{l}\tau(\mathrm{H} 105 \mathrm{C} 40 \mathrm{C} 39 \mathrm{C} 38)[15]+\tau(\mathrm{H} 106 \mathrm{C} 40 \mathrm{C} 39 \mathrm{C} 38)[16]+\tau(\mathrm{H} 107 \mathrm{C} 40 \mathrm{C} 39 \mathrm{C} 38) \\
{[16]}\end{array}$ \\
\hline 243 & 233 & & & $\tau(\mathrm{C} 38 \mathrm{C} 39 \mathrm{C} 40 \mathrm{H} 106)+\tau(\mathrm{C} 28 \mathrm{C} 29 \mathrm{C} 30 \mathrm{H} 90)+\tau(\mathrm{C} 15 \mathrm{C} 12 \mathrm{C} 14 \mathrm{H} 64)$ \\
\hline
\end{tabular}




\begin{tabular}{|c|c|c|c|}
\hline 238 & 228 & & $\tau(\mathrm{H} 65 \mathrm{C} 14 \mathrm{C} 12 \mathrm{C} 10)[12]$ \\
\hline 235 & 226 & & $\tau(\mathrm{H} 100 \mathrm{C} 37 \mathrm{~N} 35 \mathrm{C} 34)[10]+\tau(\mathrm{H} 121 \mathrm{C} 51 \mathrm{C} 50 \mathrm{C} 48)[10]$ \\
\hline 234 & 225 & & $\begin{array}{l}\tau(\mathrm{H} 101 \mathrm{C} 37 \mathrm{~N} 35 \mathrm{C} 34)[10]+\tau(\mathrm{H} 99 \mathrm{C} 37 \mathrm{~N} 35 \mathrm{C} 34)[16]+\tau(\mathrm{H} 100 \mathrm{C} 37 \mathrm{~N} 35 \mathrm{C} 34) \\
{[19]}\end{array}$ \\
\hline 232 & 223 & & $\tau(\mathrm{C} 48 \mathrm{C} 50 \mathrm{C} 51 \mathrm{H} 121)+\tau(\mathrm{C} 28 \mathrm{C} 25 \mathrm{C} 26 \mathrm{H} 84)$ \\
\hline 227 & 218 & $219 \mathrm{~m}$ & $\tau(\mathrm{H} 71 \mathrm{C} 17 \mathrm{C} 16 \mathrm{C} 15)[15]$ \\
\hline 226 & 217 & & $\tau(\mathrm{H} 111 \mathrm{C} 45 \mathrm{C} 44 \mathrm{C} 43)[13]+\tau(\mathrm{H} 112 \mathrm{C} 45 \mathrm{C} 44 \mathrm{C} 43)[12]$ \\
\hline 218 & 209 & & $\tau(\mathrm{C} 28 \mathrm{C} 29 \mathrm{C} 30 \mathrm{H} 90)+\tau(\mathrm{C} 28 \mathrm{C} 25 \mathrm{C} 26 \mathrm{H} 84)+\tau(\mathrm{C} 43 \mathrm{C} 44 \mathrm{C} 45 \mathrm{H} 111)$ \\
\hline 212 & 204 & & $\tau(\mathrm{H} 86 \mathrm{C} 26 \mathrm{C} 25 \mathrm{C} 23)[13]$ \\
\hline 205 & 197 & $196 \mathrm{~m}$ & $\delta(\mathrm{C} 23 \mathrm{C} 21 \mathrm{~N} 19)+\delta(\mathrm{C} 2 \mathrm{C} 4 \mathrm{C} 5)+\delta(\mathrm{C} 28 \mathrm{C} 25 \mathrm{C} 23)$ \\
\hline 199 & 191 & & $\delta(\mathrm{C} 8 \mathrm{C} 10 \mathrm{C} 12)+\delta(\mathrm{C} 2 \mathrm{C} 4 \mathrm{C} 5)+\delta(\mathrm{C} 28 \mathrm{C} 25 \mathrm{C} 23)$ \\
\hline 198 & 190 & & $\delta(\mathrm{O} 46 \mathrm{C} 44 \mathrm{C} 48)[28]$ \\
\hline 189 & 181 & & $\tau(\mathrm{O} 49 \mathrm{C} 48 \mathrm{C} 44 \mathrm{C} 45)$ \\
\hline 174 & 167 & & $\tau(\mathrm{C} 4 \mathrm{C} 6 \mathrm{C} 8 \mathrm{C} 9)$ \\
\hline 167 & 160 & $159 \mathrm{~m}$ & $\tau(\mathrm{C} 44 \mathrm{C} 43 \mathrm{C} 42 \mathrm{O} 52)$ \\
\hline 159 & 153 & & $\tau(\mathrm{C} 22 \mathrm{C} 21 \mathrm{~N} 19 \mathrm{C} 20)$ \\
\hline 152 & 146 & & $\delta(\mathrm{O} 1 \mathrm{C} 2 \mathrm{C} 4)[13]$ \\
\hline 143 & 137 & $138 \mathrm{~m}$ & $\gamma(\mathrm{C} 40 \mathrm{C} 38 \mathrm{O} 41 \mathrm{C} 39)[10]$ \\
\hline 133 & 128 & & $\tau(\mathrm{O} 7 \mathrm{C} 6 \mathrm{C} 4 \mathrm{C} 5)$ \\
\hline 130 & 125 & & $\delta(\mathrm{C} 34 \mathrm{~N} 35 \mathrm{C} 37)$ \\
\hline 128 & 123 & & $\gamma(\mathrm{N} 35 \mathrm{C} 32 \mathrm{C} 38 \mathrm{C} 34)[15]$ \\
\hline 125 & 120 & $122 \mathrm{~m}$ & $\tau(\mathrm{C} 23 \mathrm{C} 12 \mathrm{~N} 19 \mathrm{C} 20)$ \\
\hline 123 & 118 & & lattice \\
\hline 118 & 113 & 113 & lattice \\
\hline 115 & 110 & & lattice \\
\hline 111 & 107 & 107 & lattice \\
\hline 107 & 103 & 100 & lattice \\
\hline 96 & 92 & 95 & lattice \\
\hline 95 & 91 & 91 & lattice \\
\hline 88 & 84 & 83 & lattice \\
\hline 80 & 77 & & lattice \\
\hline 79 & 76 & 75 & lattice \\
\hline 76 & 73 & 71 & lattice \\
\hline 71 & 68 & & lattice \\
\hline 68 & 65 & 66 & lattice \\
\hline 64 & 61 & 60 & lattice \\
\hline 60 & 58 & & lattice \\
\hline 57 & 55 & 52 & lattice \\
\hline 44 & 42 & 43 & lattice \\
\hline 43 & 41 & 39 & lattice \\
\hline 34 & 33 & 36 & lattice \\
\hline
\end{tabular}

${ }^{*}$ Only PED values greater that $9 \%$ are given.

Modes without PED were visually determined.

Nomenclature: $\tau=$ torsion; $\delta$ = bending; wag. = wagging; $\gamma=$ out of plane bending; $v=$ stretching; $v_{\mathrm{as}}=$ asymmetric stretching; $\mathrm{v}_{\mathrm{s}}=$ symmetric stretching. $\mathrm{vs}=$ very strong; $\mathrm{s}=$ strong; $\mathrm{m}=$ medium; $\mathrm{w}$ = weak; $\mathrm{vw}=$ very weak. 